\title{
WISSENSCHAFTSEVALUATION: WOHIN GEHT DIE REISE?
}

\author{
STEFAN HORNBOSTEL
}

DOI: $10.22163 /$ fteval.2019.459

$\mathrm{W}$ enn man Googles Ngram Viewer Glauben schenkt, dann hat in der englischsprachigen Bücherwelt die Häufigkeit, mit der der Begriff „Evaluation“ auftaucht, Mitte der 1980er Jahre ihren Höhepunkt überschritten und sinkt seitdem, während die deutschen Beiträge noch ansteigen. Die Zahl der Zeitschriftenartikel, die sich um Evaluation drehen, steigt allerdings international - laut Web of Science - sehr kontinuierlich an. Auch wenn diese Publikationen sich natürlich nur zu einem sehr kleinen Teil mit Hochschul- und Wissenschaftsevaluation beschäftigen - denn evaluiert wird schließlich überall - stellt sich die Frage, was dieser schwer zu deutende Befund über die inzwischen ubiquitären Bewertungsprozeduren aussagt. Denn Kritik an Evaluationen, Akkreditierungen, leistungsorientierter Mittelverteilung, Qualitätssicherungssystemen, Zertifizierungen, Auditierungen, Rankings und Ratings ist weder neu, noch auf das Wissenschaftssystem beschränkt. Anstelle der pauschalen, aber Aufmerksamkeit heischenden Diagnose „Evaluitis” als neues gesellschaftliches Krankheitsbild im Allgemeinen und in der Wissenschaft im Besonderen, ist heute eine sehr viel sachlichere und konstruktivere Kritik getreten.

Dies, die Vielfalt eingesetzter Evaluationsverfahren und ihre sehr unterschiedlichen Zwecke machen es allerdings - zumindest, wenn man auf ganz Europa blickt - auch schwer eine allgemeine Einschätzung darüber zu geben, ob Evaluationspraxen überholungsbedürftig oder angemessen sind, ob sie unter dem Strich förderlich, nutzlos oder schädlich waren.

Gleichwohl lassen sich einige Trends feststellen, die offenbar in vielen Verfahren erkennbar sind:

\section{RITUALISIERUNG UND BERECHENBARKEIT}

Fast alle Evaluationsverfahren haben bei ihrer Einführung einen initialen Effekt, der auf das Verfahren selbst oder auch nur auf den Umstand, dass eine Evaluation erfolgt, zurückzuführen ist. Mit der Zahl der Iterationen wird dieser Effekt geringer, teils, weil die Evaluierten recht genau wissen, was erwartet wird und gelernt haben, sich in Szene zu setzen, teils, weil das Leistungsniveau gestiegen ist. Dies macht zweifellos die Begutachtung komplizierter, bedeutet aber nicht unbedingt, dass Evaluationen zur sinnentleerten Routine werden. Rituale sind durchaus Formen der gemeinschaftlichen Selbstvergewisserung, die helfen können auch kritische und komplexe Konstellationen in einer einheitsstiftenden
Weise behandelbar zu machen. Das setzt allerdings eine gewisse Ernsthaftigkeit (Evaluationskultur) voraus und ein Verfahren, das die diskursive und kritische Verarbeitung der Ergebnisse möglich macht.

\section{CONTROLLING, OM, AUDIT, MONITORING, BERATUNG, STRATEGISCHE PLANUNG}

Wenn man Evaluation im weitesten Sinne als systematische Untersuchung der Erreichung selbstgesetzter oder normativ vorgegebener Ziele versteht, dann sind die Grenzen zwischen den verschiedenen Verfahren relativ unscharf. Auf anekdotischer Evidenz beruhend, scheint sich ein Trend "individualisierter Verfahren“ abzuzeichnen, bei denen - häufig anlassbezogen - strategische Beratung im Vordergrund steht. Aber auch Evaluationen im engeren Sinne (und ebenso Akkreditierungen) werden zunehmend in einem für die lokalen Verhältnisse maßgeschneiderten Format von den Lehr- und Forschungseinrichtungen selbst durchgeführt und als Qualitätssicherung in die institutionelle Governance integriert. Das ersetzt zwar externe Evaluationen nicht, erhöht aber die Resilienz der Evaluierten deutlich.

\section{METRIK ODER PEER-REVIEW?}

Die wachsende Informiertheit der Evaluierten hat auch mit der Verbreitung von Informationssystemen zu tun, die in elaborierter Form eine Fülle von Informationen prozessproduziert bereitstellen. Dabei ist die Frage, ob ein tendenziell fehleranfälliges und konservatives Peer-Review oder eine kontextfreie, für Fehlinterpretationen anfällig, Metrik die bessere Lösung ist, vermutlich falsch gestellt. Die wachsende Fähigkeit auch textuelle Informationen zu analysieren, wird in Zukunft wahrscheinlich den Algorithmen größere Bedeutung verschaffen. Darüber hinaus erlauben Standardisierungen (wie sie in Deutschland mit dem Kerndatensatz Forschung eingeführt wurden) eine skalierbare Möglichkeit neben den eigenen Informationsbedürfnissen in einem selbstbestimmten Rahmen externe Referenzgrößen (etwa über Benchmarking) einzubeziehen. Gut möglich, dass die Peers in Zukunft stärker in der strategischen Beratung gefragt sind, als in der Evaluation. 


\section{WISSENSCHAFTSINTERNE KRITERIEN ODER GESELLSCHAFTLICHER IMPACT?}

Auch die Bewertungskriterien sind in Bewegung geraten. Immer stärker wird nach dem wissenschaftsexternen Impact von Forschung und Lehre gefragt. Dabei geht es nicht mehr um einfache ökonomische Effekte, sondern in einem sehr umfassenden Sinne um gesellschaftliche Veränderungen, die durch Wissenschaft induziert werden. Gegenwärtig existieren allerdings kaum solide Mess- oder Bewertungswerkzeuge, um derartigen Impact (der sich zudem in sehr unterschiedlichen zeitlichen Horizonten entfaltet) zu erheben. Der Typus von Forschung, um den es dabei geht, ist vermutlich mit dem Begriff der "Translation" besser beschrieben, als mit dem alten Konzept der "Anwendungsorientierung".

\section{TRANSAKTIONSKOSTEN}

Der mit Evaluationen verbundene Aufwand ist erheblich. Die Frage, inwieweit Evaluationen positive Wirkungen entfalten und wieviel Evaluation mit welchen Kosten angemessen ist, ist daher notwendig und legitim. Allerdings verschiebt sich mit der open science Bewegung auch die Bewertung der Transaktionskosten möglicherweise weg von einer rein ökonomischen Betrachtung hin zu einer eher durch Informationsmanagement und Qualitätssicherung geprägten Sichtweise.

Wissenschaftsevaluationen werden also vermutlich nicht aussterben, sich aber möglicherweise erheblich verändern und mit anderen Prozessen amalgamieren. Dabei wird eine elaborierte institutionelle Selbststeuerung wohl auch in Zukunft von einer extern organisierten Evaluation begleitet werden. Das allerdings wird ein höheres Maß an Professionalisierung der Peers notwendig machen.

\section{AUTOR}

Prof. Dr. Stefan Hornbostel

Deutsches Zentrum für Hochschul- und Wissenschaftsforschung GmbH (DZHW)

Schützenstraße 6a, 10117 Berlin

E: hornbostel@dzhw.eu 